\title{
Gold Chemistry Reported at the RSC Dalton Discussion Meeting on 'Metal Clusters'
}

\author{
David Thompson \\ Consulting Chemist, Reading, England, UK
}

\begin{abstract}
This meeting was organised by the Dalton Division of the Royal Society of Chemistry at the University of Southampton from 3-5 January 1996 along the lines of the well established 'Faraday Discussions', and accordingly was called 'Dalton Discussion No 1'. Abstracts of presentations were distributed beforehand and this and the open attitude apparent in the oral presentations helped to stimulate substantial and lively discussions after both the introductory review lectures and groups of small presentations on experimental work.
\end{abstract}

The first talk was entitled 'Gold - Flexible Friend in Cluster Chemistry' and was given by Professor Michael Mingos (Imperial College, London) (1). He opened by indicating that gold had proved to be remarkably attractive to mankind from earliest times. Even though our greater understanding of its properties has removed much of the mystical and occult connotations, gold retains a special place in our cultures. The origins of modern chemistry are also closely allied with early attempts to convert base metals into gold, an early example of research targeted at wealth creation! Professor Mingos described the flexible metal bonding modes entered into by gold, which enable it to form a wide range of polyhedral molecules, and also make it an important component in supramolecular chemistry.

In the gas phase, gold forms stable dimeric molecules, $\mathrm{Au}_{2}$, which have a dissociation energy of $228 \mathrm{~kJ}$ per mole and a bond length of $2.472 \AA$. The element itself has a heat of atomization of $368 \mathrm{~kJ}$ per mole and a metal-metal distance in the cubic close-packed lattice of $2.884 \AA$. Although these energies are smaller than those for metals occurring earlier in the transition series, they are sufficient for gold to form strong metal-metal bonds in a wide range of compounds. These strong bonds allow gold to form cluster compounds stabilised by phosphine ligands in which gold has an intermediate oxidation state between $(+1)$ and 0 . Gold is also able to form cluster compounds with a wide range of other metals. In the $(+2)$ oxidation state, gold forms a more limited range of principally dinuclear species supported by additional bridging ligands.
Gold, and especially the $\mathrm{Au}\left(\mathrm{PPh}_{3}\right)$ fragment, has a unique ability to form hypervalent compounds of the main group elements such as $\left[\mathrm{C}\left\{\mathrm{Au}\left(\mathrm{PPh}_{3}\right)\right\}_{6}\right]^{2+}(\mathrm{I})$ and $\left[\mathrm{N}\left\{\mathrm{Au}\left(\mathrm{PPh}_{3}\right)\right\}_{5}\right]^{2+}(2)$. In these hypervalent compounds the gold-gold interatomic distances are ca $3.00 \AA$ which is significantly longer than the bond length in the metal $(2.884 \AA)$. These longer bonds are individually weak but cumulatively could contribute to the overall stability of the molecule because of the large number of such interactions. Thus in the octahedral Compound (I) there are twelve interactions of this type.

In many gold(I) compounds the geometry about the metal is linear, but there are additional contacts with other gold atoms which are significantly longer than the metal-metal distance found in the metal $(2.884 \AA)$, but shorter than the sum of the estimated van der Waals radii of gold $(3.60 \AA)$. It has been suggested that these bonds are analogous to hydrogen bonds (3), or S...S or halogen-halogen intermolecular interactions (4).

Thus gold has a remarkable ability to form metalmetal bonds with a wide range of strengths, and examples to illustrate this phenomenon were provided. If the metal-metal bond length approaches the distance found in $\mathrm{Au}_{2}(2.47 \AA)$, its strength must be ca $200 \mathrm{~kJ}$ per mole, but in cluster compounds where the metalmetal bond lengths approximate to those found in the bulk metal $(2.884 \AA)$ then each metal-metal bond will be associated with ca $60-100 \mathrm{~kJ}$ per mole, and in those compounds where the metal-metal contacts are 
$3.0-3.2 \AA$ each of these interactions probably contributes $10-30 \mathrm{~kJ}$ per mole. The interaction will be energetically insignificant only when the metal-metal

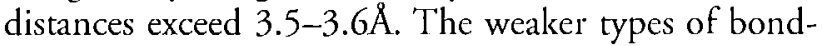
ing were likened to the comparatively weak hydrogen bonds well known in other branches of chemistry.

Many gold(I) phosphine complexes have been reported and these are used as reagents in organometallic and cluster chemistry, but the chemistry of gold(I) with nitrogen ligands has been neglected (1). Mingos $e t$ al have recently synthesised, isolated, and spectroscopically characterized salts of $\left[\mathrm{Ag}\left(\mathrm{NH}_{3}\right)_{2}\right]^{+}$, previously only reported in solution, and $\left[\mathrm{Ag}\left(\mathrm{NH}_{2} \mathrm{R}\right)_{2}\right]^{+}$; and determined the structure of $\left[\mathrm{Au}\left(\mathrm{NH}_{3}\right)_{2}\right] \mathrm{Br}$ (see Figure 1).

There is considerable interest in the possibility of

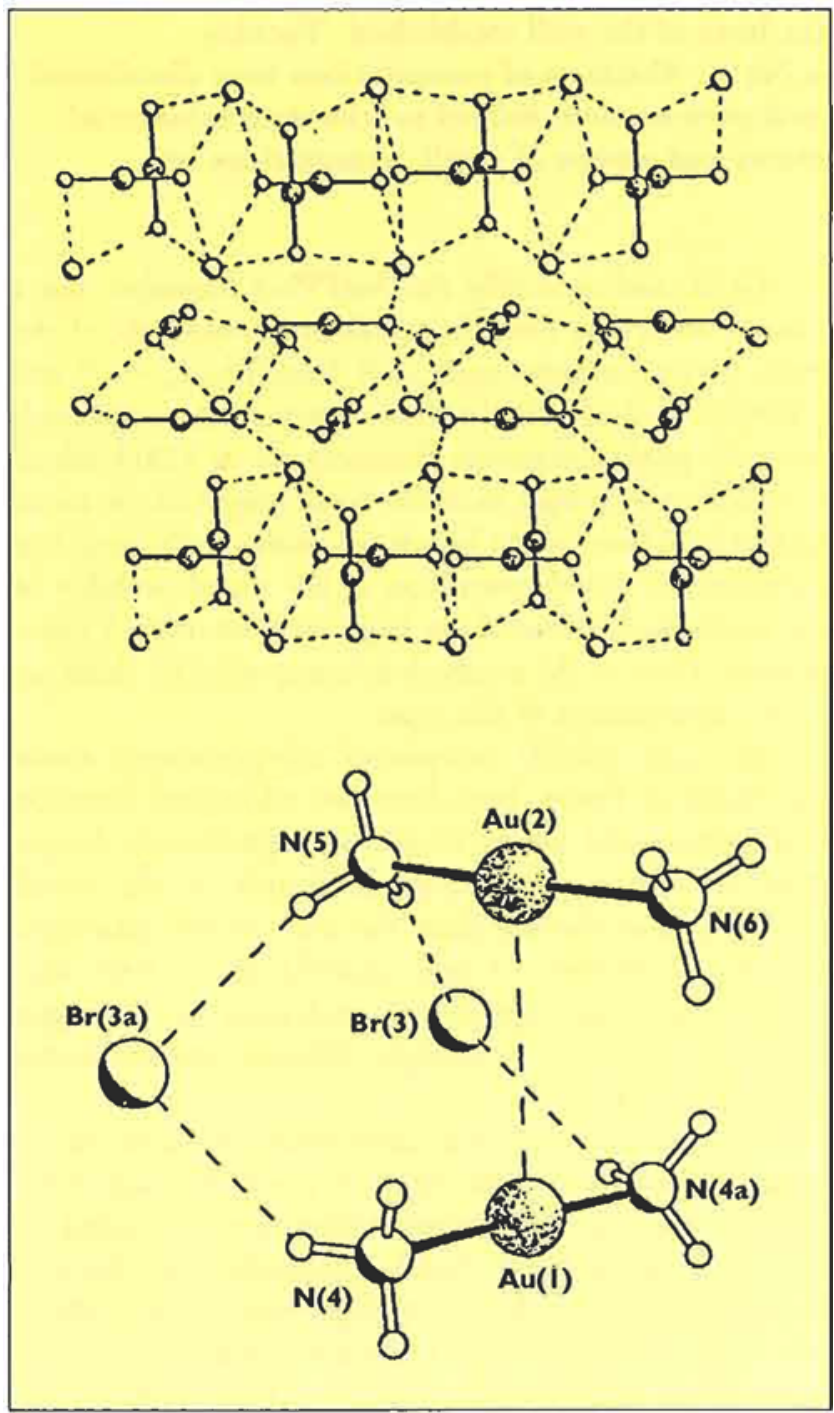

Figure 1 Structure of $\left[\mathrm{Au}\left(\mathrm{NH}_{3}\right)_{2}\right] \mathrm{Br}$. An interaction between a pair of gold atoms is shown at the bottom and the network of hydrogen bonds linking the ions at the top. using hydrogen-bonding types of interactions for designing the structures of molecular solids. Crystal engineering of this type could be particularly important for the design of materials which might display superior non-linear optical or magnetic properties. The work reported to date on gold(I) compounds suggests that it may be possible to use the analogy with hydrogen bonding to construct infinite solids where the predominant gold-gold intermolecular interactions could lead to predictable supramolecular structures.

The coloured gold colloids first synthesised by Michael Faraday were the forerunners of clusters, and the colour of the colloids varied with the size of the particles. The cluster compounds of gold are generally stabilised by tertiary phosphines (1) and the first examples were described by Malatesta in the 1960s (5). Cluster compounds of gold with 4 to 13 metal atoms are now well established and those with more than seven have an interstitial gold atom located in the centre of the cluster (1). The simplest clusters have a tetrahedron of metal atoms, either with no bridging ligands, e.g. $\left[\mathrm{Au}_{4}\left(\mathrm{PBu}_{3}\right)_{4}\right]^{2+}$, or with bridging ligands on two of the edges, e.g. $\left[\mathrm{Au}_{4}(\mu-\mathrm{I})_{2}\left(\mathrm{PPh}_{3}\right)_{4}\right]$.

Compounds described by Schmidbaur $(1,2)$ containing interstitial main group elements such as $\mathrm{C}, \mathrm{N}$, $\mathrm{O}, \mathrm{P}$ or $\mathrm{S}$ may be formulated as cluster compounds or molecular coordination compounds of the main group atoms with the $\mathrm{AuPR}_{3}$ radical bonded to the central atom in a manner analogous to that for $\mathrm{H}$ and $\mathrm{CH}_{3}$. This formulation is supported by the observation that the majority of these compounds have $\mathrm{Au}-\mathrm{Au}$ distances which are approximately $3.00 \AA$. These distances are longer than are found either in the bulk metal or in the majority of gold cluster compounds, and are closer to the bond lengths found in those linear gold(I) compounds which show intermolecular interactions. The structures of some of these compounds is indicated in Figure 2.

Amongst other presentations illustrating recent advances in gold chemistry was one by Catherine Housecroft (Institute for Inorganic Chemistry, Basel, Switzerland) (6), describing cluster core geometrical variation in heterometallic boride clusters containing $\mathrm{RhRu}_{4}$ skeletons such as $\left[\mathrm{RhRu}_{4} \mathrm{H}(\mathrm{nbd})(\mathrm{CO})_{12} \mathrm{~B}\right.$ $\left.\left(\mathrm{AuPPh}_{3}\right)\right]$ (II) (nbd = norbornadiene) with a squarebased pyramidal $\mathrm{RhRu}_{4}$ framework, the reaction of (II) with carbon monoxide giving $\left[\mathrm{RhRu}_{4} \mathrm{H}(\mathrm{CO})_{14} \mathrm{~B}\right.$ $\left(\mathrm{AuPPh}_{3}\right)$ ]; and Ian Salter (University of Exeter) (7), who described variable temperature NMR studies on mixed metal cluster compounds containing gold. The $\mathrm{X}$-ray crystal structures of $\left[\mathrm{Au}_{2} \mathrm{Ru}_{4}\left(\mu_{3}-\mathrm{H}\right)(\mu-\mathrm{H})(\mu\right.$ $\left.\mathrm{dppf})(\mathrm{CO})_{12}\right]$ and $\left[\mathrm{AuCuRu}_{4}\left(\mu_{3}-\mathrm{H}\right)_{2}(\mu-\mathrm{dppf})(\mathrm{CO})_{12}\right]$ 


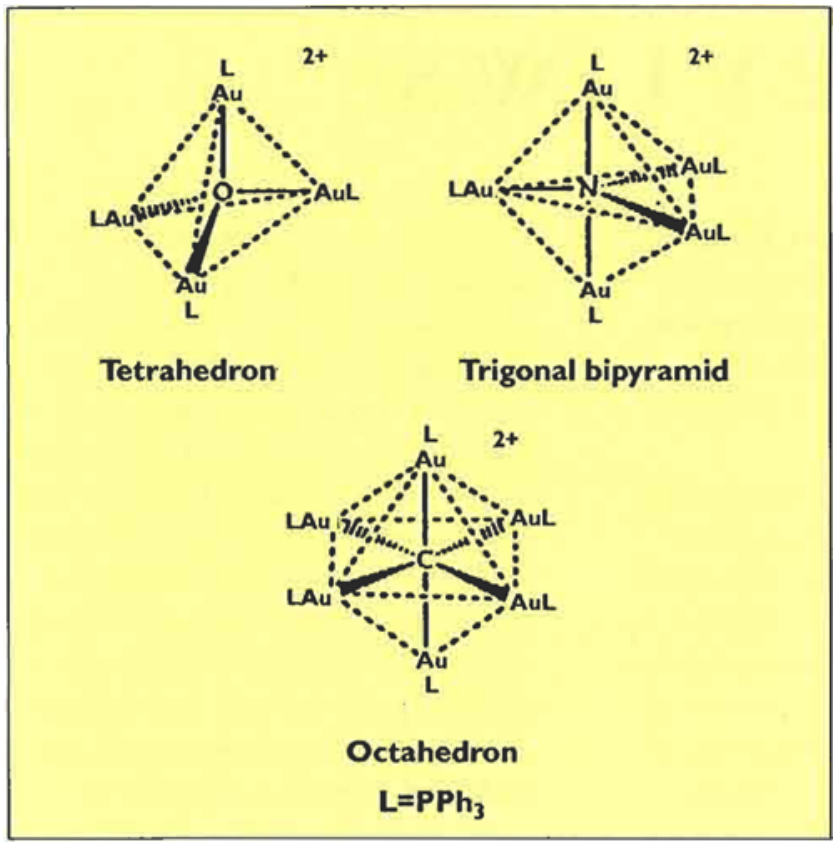

Figure 2 Examples of gold cluster compounds with interstitial main-group atoms. These compounds may also be considered to be molecular co-ordination compounds of the main-group element with $A u\left(P R_{3}\right)$ radicals.

(dppf $=1,1^{-}$-bis(diphenylphosphino)ferrocene), revealing a variety of metal-metal bonding distances involving gold. Fluxional processes in solution were studied for these and related complexes.

Robin Whyman (University of Liverpool) (8) described the preparation using vapour synthesis techniques, characterization, and properties of solvent-stabilized metal nanoparticles for metals from Groups 8$10(\overline{\mathrm{VII}})$ and $11(\mathrm{IB})$. The behaviour of the $1-3 \mathrm{~nm}$ sized gold nanoparticles was unique - they exhibited unusual time and concentration-dependent behaviour. A full description of this work is given elsewhere in this issue of Gold Bulletin (9).

Amongst the posters, presented at an evening session, was one by A. M. R. Gibson and G. Reid of the University of Southampton entitled 'Mono- and Di-
Nuclear complexes of Phospha-Thia Ligands with Gold(I)'. Mononuclear Au(I) complexes of three linear $\mathrm{P}_{2} \mathrm{~S}_{2}$ donors, $[\mathrm{Au}(\mathrm{L})] \mathrm{PF}_{6}\left(\mathrm{~L}=\mathrm{P}_{2} \mathrm{~S}_{2}\left\{\mathrm{C}_{2}\right\}, \mathrm{P}_{2} \mathrm{~S}_{2}\left\{\mathrm{C}_{3}\right\}\right.$, or $\left.\mathrm{P}_{2} \mathrm{~S}_{2}\{\mathrm{o}-\mathrm{Ph}\}\right)$ have been prepared by the reaction of AuCl(tht) (tht $=$ tetrahydrothiophene) with $\mathrm{L}$ and $\mathrm{T} \mathrm{PF}_{6}$ in acetonitrile solution. The non-conducting dinuclear species $\left[\mathrm{Au}_{2} \mathrm{Cl}_{2}(\mathrm{~L})\right]$ are obtained by the reaction of $\mathrm{AuCl}(\mathrm{tht})$ with $\mathrm{L}$ in a $2: 1$ molar ratio in dichloromethane solution.

This reviewer suggests that now that an interest in catalysis by gold systems is reawakening (10), the relationships between gold colloids, and gold and mixed-metal gold clusters, and the ability of such species to catalyse chemical transformations in their own right and when supported on high surface area oxides may prove to be productive areas for investigation.

\section{REFERENCES}

1 D.M.P. Mingos, J. Chem, Soc., Dalton Trans., 1996,561

2 H. Schmidbaur, Pure Appl. Chem., 1993, 65, 691

3 H. Schmidbaur, W. Graf and G. Muller, Angew. Chem., Int. Ed. Engl., 1988, 27, 417

4 S.S. Pathenini and G.R. Desiraju, J. Chem. Soc., Dalton Trans., 1993, 319

5 L. Malatesta, Chem. Commun., 1965, 212.

6 A.D. Hattersley, C.E. Housecroft and A.L. Reingold, J. Chem. Soc., Dalton Trans., 1996, 603

7 I.D. Salter, V. Sik, S.A. Williams and T. Adatia, J. Chem. Soc., Dalton Trans., 1996, 643

8 R.W. Devenish, T. Goulding, B.T. Heaton and R. Whyman, J. Chem. Soc., Dalton Trans., 1996, 673

9 R. Whyman, Gold Bull., 1996, 29, 11.

10 M. Haruta, S. Tsubota, T. Koboyashi, H. Kageyama, M.J. Denet and B. Delmon, J. Catal., 1993, 144, 175 